\title{
MQTT Messages-An Overview
}

\author{
Dr. S. Thavamani ${ }^{1}$, U. Sinthuja ${ }^{2,3}$ \\ ${ }^{1}$ Associate Professor, Department of Computer Application, Sri Ramakrishna College of Arts and Science, Coimbatore, \\ Tamilnadu \\ ${ }^{2}$ Research Scholar, Department of Computer Science, Sri Ramakrishna College of Arts and Science, Coimbatore, Tamilnadu \\ ${ }^{3}$ Assistant Professor, Department of Information Technology, Hindusthan College of Arts and Science, Coimbatore, Tamilnadu
}

\begin{tabular}{ll}
\hline ARTICLE INFO & ABSTRACT \\
\hline Published Online: & $\begin{array}{l}\text { This research work greatly concerning the trending Protocol known as Message Queue Telemetry } \\
\text { 23 April 2021 }\end{array}$ \\
& $\begin{array}{l}\text { Transport (MQTT) which is used by Internet of Things (IoT) to pass messages by both ends. The } \\
\text { communication between the huge amounts of devices is enabled by IPv6 and light weight } \\
\text { communication protocols such as MQTT. The goal was to develop a protocol which is bandwidth- } \\
\text { efficient and uses little battery power. Most of the present-day surveys focusing on IoT MQTT } \\
\text { protocol protection. Even though security is very much concerned, it is also important to }\end{array}$ \\
Corresponding Author: & $\begin{array}{l}\text { concentrate on MQTT messages with its types, structure and so on. } \\
\text { Dr. S. Thavamani }\end{array}$
\end{tabular}

KEYWORDS: Internet of Things, MQTT Protocol

\section{INTRODUCTION}

The prevalence of embedded devices with their very own memory and computational power, as well as the ability to connect with each other, has given rise to new attack vectors that have proven difficult to protect against. According to [1], interconnected devices such as sensors, appliances, and cameras represent some of the components of this network, which was named the Internet of Things (IoT) by Kevin Ashton at a conference in 1999. The IoT became possible by the sudden increase in smart devices that manufacturers developed and released on the market. This was accomplished without having properly considered all aspects of security and device limitations. It is assumed that by the year 2020, most of the devices that the consumer will have access to will be able to connect to the Internet. Kevin also stated that most of the information available right now was recorded using different conventional methods.

\section{THE MESSAGE QUEUE TELEMETRY TRANSPORT (MQTT) PROTOCOL}

The protocol was created by IBM as a machine-to-machine, lightweight communication method. MQTT was standardized by ISO/IEC 20922 and was further accepted as part of OASIS. At its core, MQTT is a messaging protocol that uses the publish-subscribe communication model, where the clients themselves do not require updates, thus in turn causing a reduction of used resources, which makes this model optimal for use in a low-bandwidth environment. Furthermore, the protocol functions on a server-client system where the server, called a broker, pushes updates to MQTT clients. The clients won't send messages directly to each other, instead relying on the broker for this. Every MQTT message contains a topic, organized in a tree-like structure, to which the clients can subscribe or publish. The broker receives published messages from clients that contain a certain value or command and relays the information to every client that has subscribed to that specific topic. As can be seen, the MQTT protocol was designed for asynchronous communication, where subscriptions or publishing to or from different entities take place in a parallel order. The protocol is also able to provide reliable transfers by choosing between three types of reliability mechanism, also called Quality of Service (QoS).[2]

When compared to other protocols like HTTP, the MQTT protocol has a considerably smaller footprint, making MQTT, as stated above, much more suitable for resourceconstrained environments. Although the MQTT protocol has many advantages, not every MQTT-based broker has similar or comparable abilities for entity authentication or encryption. Eclipse's open-source application, called Mosquitto, is able to provide most of standardized features of the MQTT protocol, such as SSL/TLS and client certificate support. The Mosquitto broker, by default, does not provide security for its messaging scheme and 
authentication information is sent in plaintext; therefore, it requires security mechanisms to protect the transferred information.[3]

\section{REAL-WORLD APPLICATIONS}

The few of the applications has discussed here to show the importance of MQTT Protocol in real-time applications

- It is employed in the Facebook Messenger application. The company chose MQTT because of its specific design for applications such as sending telemetry data to and from space probes, which requires less bandwidth and battery power. The company was able to achieve phone-to-phone communication by maintaining MQTT delivery in hundreds of milliseconds.

- LAMA (Location Aware Messaging for Accessibility) is a system which makes essential information to interests of the people and area accessible to each other. Smart Phones, MQTT and WebSphere Message Broker, and even some smart software applications, all are used in the system.

- GAIAN Database- A distributed federated database written in Java that uses a biologically inspired self-organization principle to minimise management. GaianDB has already been used in large, distributed systems. queries for semantic joins in text analytics applications and has piqued the interest of key customers in the armed forces.

- The Open Geospatial Consortium Sensor Things API standard specification has an MQTT extension in the standard as an additional message protocol binding. It was demonstrated in a US Department of Homeland Security IoT Pilot.[4]

\section{MQTT CLIENT AND BROKER}

Any device (from a microcontroller to a full-fledged server) that runs a MQTT library and connects to a MQTT broker over a network is considered a MQTT client. The MQTT client, for example, can be a very small, resourceconstrained device that connects via a wireless network and has a bare-bones library. For testing purposes, the MQTT client can also be a standard computer running a graphical MQTT client.
MaTT Message Publising Message Flow

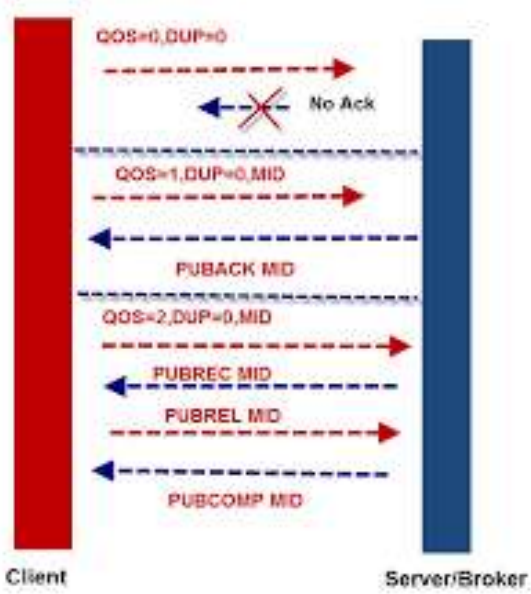

Figure 1. MQTT Message Flow

The broker functions as a post office; MQTT does not use the intended recipient's address but instead uses the subject line "Topic," and anyone who wants a copy of that message will subscribe to that topic. A single broker's message can be delivered to multiple clients (one to many capability). Similarly, multiple publishers can distribute topics to a single subscriber (many to one). Each client can produce and receive data by publishing and subscribing, i.e., devices can publish sensor data while still receiving configuration information or control commands (MQTT is a bi-directional communication protocol). This facilitates data sharing as well as device management and control.

The following are the primary benefits of using a MQTT broker:

- Client connections are no longer vulnerable or insecure.

- Scalable from a single system to hundreds

- All client connection states, including security credentials and certificates, are managed and tracked.

- Reduced network strain without jeopardising network security (cellular or satellite network)

\section{CONCEPTS IN MQTT MESSAGES}

A. Publish/subscribe: In MQTT protocol, publisher publishing messages and users subscribing to topics that are commonly considered as a Publish/Subscribe model [7]. Subscriber subscribes to particular topics which are relate to them and by that receive every message are published to those topics [8]. On the other hand, clients can publish messages to topics, in such a way that allow all subscribers to access messages of those topics.

B. Topics and subscriptions: In MQTT, publisher publish messages to topics that can be considered as message subject. Subscriber, thus, subscribe to topics to get specific messages. The Subscriptions of topics can be express, that restricts the data which are collect to the particular topic [7]. 
Fixed header present in all MQTT Control Packets

Variable header present in some MQTT Control

Packets Payload present in some MQTT Control Packets

C. QoS: A quality-of-service metric can be specified for each connection to the broker. These are listed in ascending order of overhead:

- At most once - the message is sent only once, and neither the client nor the broker takes any additional steps to acknowledge receipt (fire and forget).

- At least once - the sender retries the message several times until an acknowledgement is received (acknowledged delivery).

- Exactly once - the sender and receiver engage in a two-level handshake to ensure that only one copy of the message is received (assured delivery).

[5] This field has no effect on how the underlying TCP data transmissions are handled; it is only used between MQTT senders and receivers.

\section{MQTT PACKET FORMAT}

For communication, it exchanges a range of control packets in a specify manner. There are fourteen control packets in total. As shown in Figure 2, each consists of three parts.[6].

Figure 2. Common Control Packet format

A. Types of MQTT Control Packets

- CONNECT - Fixed Header / Variable Header / Payload - MQTT Client requests a connection to a Broker.

- CONNACK - Fixed Header - Acknowledge connection request.

- PUBLISH - Fixed Header / Variable Header - Publish message.

- SUBACK - Fixed Header / Variable Header / Payload - Subscribe acknowledgement.

- UNSUBSCRIBE - Fixed Header / Variable Header / Payload - Unsubscribe from a topic.

- UNSUBACK - Fixed Header / Variable Header / Payload - Unsubscribe acknowledgement.

- DISCONNECT - Fixed Header - Disconnect notification.

- PUBREL - Fixed Header / Variable Header - Publish release(QoS 2 publish received).

- PUBACK - Fixed Header / Variable Header - Publish acknowledgement.

- PUBREC - Fixed Header / Variable Header - Publish received(QoS 2 publish received).

- PUBCOMP - Fixed Header / Variable Header Publish complete.

- SUBSCRIBE - Fixed Header / Variable Header / Payload - Subscribes to a topic.

- PINGREQ - Fixed Header - PING request.

\section{B. MQTT Packet Sizes}

The fixed header field consists of the control field, and the variable header contains the packet length field. The minimum size of a packet length field is 1 byte, which is for messages less than 127 bytes.

The maximum packet size is $256 \mathrm{MB}$. The minimum packet size is only 2 bytes with a single control field and a single packet length field. Smaller packets less than 127 bytes have a 1-byte packet length field. The Packets greater than 127 and less than 16383 use 2 bytes, and so on. 7-bits are used with the 8 th bit is the continuation bit.

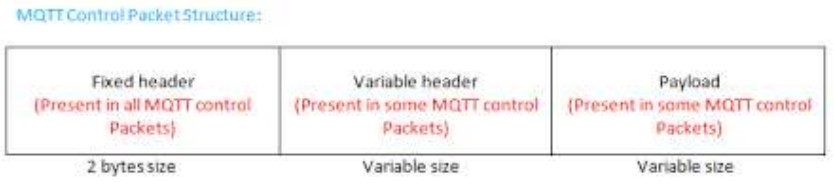

Figure-3. MQTT packet Size

\section{CONCLUSION}

The MQTT (Message Queue Telemetry Transport) protocol is a simple publish/subscribe messaging protocol. It's a common protocol for small devices. It is a free and open standard that is better suited to constrained environments than HTTP. Messages are published, and topics are subscribed to. Several clients/processes connect to a broker/monitor and subscribe to topics of interest to them. The broker and MQTT serve as a straightforward, universal interface for connecting everything. It is a text-based wire protocol designed for $\mathrm{M} 2 \mathrm{M}$ connectivity that enables the transfer of telemetry-style data in the form of messages from devices to a server or small message broker over high latency or constrained networks. Sensors and actuators, mobile phones, embedded systems in vehicles, laptops, and full-fledged computers are all examples of devices. It is extremely simple to use and supports publish-and-subscribe style communications. MQTT (Message Queuing Telemetry Transport) collects data from devices. Its purpose is to collect data from a large number of devices and transport it to a Data Centre.

\section{REFERENCES}

1. Gupta, A. IoT Hackers Handbook; AttifyInc: Sunnyvale, CA, USA, 2017.

2. Nastase, L. Security in the Internet of Things: A Survey on Application Layer Protocols. In Proceedings of the 2017 21st International Conference on Control Systems and Computer Science, Bucharest, Romania, 29-31 May 2017.

3. Dan Dinculeană and Xiaochun Cheng, Vulnerabilities and Limitations of MQTT Protocol Used between IoT Devices, Appl. Sci. 2019, 9, 848; doi:10.3390/app9050848.

4. Reginald (January 25, 2016). "S\&T's Internet of Things Pilot Demonstrates 'State of the Practical'". dhs.gov. p. 1. 
5. "IBM Knowledge Center - IBM MQ - Using MQTT with IBM Integration Bus - Quality of service and connection management".

www.ibm.com.

6. Banks, A., \& Gupta, R. (2014). MQTT Version 3.1. 1. OASIS standard.

7. Lampkin, V., Leong, W. T., Olivera, L., Rawat, S., Subrahmanyam, N., Xiang, R., ... \& Locke, D. (2012). Building smarter planet solutions with mqtt and ibm websphere mq telemetry. IBM Redbooks.

8. Hunkeler, U., Truong, H. L., \& Stanford-Clark, A. (2008, January). MQTT-S-A publish/subscribe protocol for Wireless Sensor Networks. In Communication systems software and middleware and workshops, 2008. comsware 2008. 3rd international conference on (pp. 791- 798). IEEE. 